\title{
Editorial Principles
}

The manuscript tradition was analyzed with respect to the number of identified cases of omissions, additions, variants, and obvious scribal errors as well as the sense of the text. The outcomes of this examination indicated that manuscript A should be adopted as the basis of the edition. Eight out of nine manuscripts containing question 3, namely ABDFGIKL, have been fully collated. One of the Parisian manuscripts $(\mathrm{H})$ has been collated partially. Nevertheless, it has been excluded from the apparatus because of its poor quality: low textual cohesion, abbreviations, and contaminations with idiosyncratic variants.

The present edition largely adheres to the text of A. There are several arguments to support this choice. First, the text of $A$ is preferred due to its superior textual quality in comparison with all the other manuscripts that contain question 3. It has a relatively small number of accidents in general, and in particular of the omissions of more than one word requisite for the correct understanding of the text. It also has the smallest number of variant readings or additions of more than one word. Secondly, in terms of sense and consistency, A preserves the best copy of the text, inasmuch as it contains the fewest instances of various text 'contaminations', such as scribal corrections of the meaning, added explications, or scribal mistakes. Lastly, it remains the only manuscript that features the complete text of all eight questions. Given this, the reading of $\mathrm{A}$ is also followed where it is less elegant syntactically than other witnesses.

The texts of B \& of K are also of good quality. There are two reasons why neither of them serves as the leading manuscript for this edition. Firstly, B includes extensive marginal corrections and a number of uncorrected omissions, which suggests that it did not preserve the whole text and was corrected from another witness, and $\mathrm{K}$ has extensive additions in the margins that do not appear in any other surviving witness of question 3. Secondly, they both preserve incomplete versions of the text: B contains questions 1-4 and fragments of questions 5,7 , and 8 , while $\mathrm{K}$ contains only the first four questions and a fragment of question 7 .

As to the variants, omissions, and additions of A, they have all been included in the apparatus. All the isolated accidents of other manuscripts have been ignored, unless they are meaningful for the text. All of them have been retained, however, if shared by at least two of the witnesses preserving question 3. Also, the words deleted by the scribe have been ignored. The same rule has been applied to the passages of the text labeled as deleted ('va ... cat') by the scribe, as well as to the additional explanatory titles, headlines, and numbering or sum-

The research on this volume and publication of this study in Open Access have been supported financially by the Polish National Agency for Academic Exchange (Mieczysław Bekker Programme, project number 
maries of the passages added by scribes in the margins, which have all been excluded from the apparatus criticus. Longer omissions and additions (of two words or more) have been included in the apparatus criticus.

There are some exceptions to these principles.

1) Wherever the text of A is deemed wrong or improbable, either B or K is followed.

2) In the rare cases where none of the readings of $A B K$ seems to make sense, readings from other manuscripts have been taken into account.

3) However, if none of them offers a comprehensible reading, a probable conjecture is occasionally provided, and the readings of all the manuscripts that preserve the passage are supplied in the critical apparatus.

4) In several instances of quotes, where the text is incomprehensible because the manuscripts vary and none of the readings is correct, the quotes are corrected based on modern critical editions of the sources.

The critical apparatus abides by the following principles:

1) Variants such as listed below have been excluded: iste/ille, cum/quando, vel/sive/aut/seu, nec/neque, igitur/ergo, ab/a/ex, per/secundum, etiam/et, scilicet/videlicet.

2) Omissions such as listed below have been excluded: et, etiam, etc., sic, est (especially in cases such as 'consequens/antecedens est falsum'), vel, iste/ista, ille/illa (especially in cases such as 'ista consequentia non valet'), quod, quia, tunc, igitur, ergo, igitur etc, per, secundum, in (ablativus temporis), videlicet, scilicet, item, de, ex, quam, cum, sed, ibi, ubi.

3) Additions such as listed below have been excluded:

et, etiam, etc., sic, est, vel, ille/illa, iste/ista, quod, quia, igitur, ergo, igitur etc, in (ablativus temporis), videlicet, scilicet, item, de, ex, quam, cum, sed, ibi, ubi.

However, all of such variants, omissions, and additions $(1,2,3)$ have been included in the critical apparatus if they occur in at least two of the manuscripts or appear significant for the understanding of the text.

Spelling differences, such as 'intelligere'/'intellegere' or 'saltim'/'saltem', as well as the corrections of the text in the margins, have been excluded from the apparatus criticus. If marginal notes are additions to the texts, they have been retained in the critical apparatus. Since Kilvington's work is highly technical, sometimes a change in word order (in phrases of three or more words) results 
in a change of meaning. Therefore, all such changes have been marked in the apparatus criticus. Isolated inversions have been kept in the critical apparatus only if they provide an alternative reading; all inversions have been kept, however, if shared by at least two manuscripts. Isolated accidents of conjunctions, propositions, and pronouns have been excluded from the critical apparatus unless they provide an alternative reading. This rule has been applied especially to the cases of scribal mistakes or mere negligence (for example in the cases of cum/tamen, or quod/quia, which were notoriously copied incorrectly in $G$ ). Isolated iterations of one word and omissions of the number referring to chapters/books, etc. of the works cited have also been excluded from the critical apparatus.

Certain mannerisms of Kilvington's style should be noted at this point.

1. Kilvington tends to furnish his sentences with several different linking words, which seem either redundant or simply stylistically incorrect. Since I have not identified any purpose for adding extra linking words other than just emphasis, they have in most cases been excluded from the main text and marked in the apparatus, provided that at least one manuscript offers such a reading. Some examples of this peculiarity are listed below and bolded.

Corpus quantum esset capax intensive infiniti, si esset. Sed quantitas nihil facit ad capacitatem itaque maiorem vel minorem quae est respectu intensionis in forma (...).

Posito tamen, gratia exempli, quod angelus et B grave forent aequalis potentiae, non sequitur: in infinitum isti sunt aequalis potentiae, igitur neutrum potest $\langle$ agere $\rangle$ in aliud quia, ut patet per Ricardum De Trinitate parte 4 capitulo ultimo (...).

Exponens illud Genesis III: "collocavit Deus ante paradisum Cherubin, et flammeum gladium," ubi dicit Magister quod (...).

In a few instances, if all the manuscripts (or all but one) preserve the additional linking word, it has been kept to convey the character of the text.

Tunc Deus punit Platonem plus quam Socratem per unum diem poena damni et poena sensus, et posset rationabiliter tantum punire Socratem, si vellet, sicut Platonem, cum aequaliter peccaverint, igitur posset rationabiliter Deus addere poenae Socrati tantam poenam sen- 
sus quanta aequaretur poenae Platonis per primum diem; et talis, ut argutum est, foret poena infinita vel quantumcumque vis magna in gradu, igitur etc.

2. Throughout his work, Kilvington's attribution of grammatical gender to the word dies inconsistently oscillates between masculine and feminine, which was a common medieval practice. In the edition, the usage is unified, and masculine is consistently used, with the variants retained in the critical apparatus. The same rule is applied to punctum/punctus, which is treated as neuter in the edition, though appearing either as neuter or as masculine in Kilvington's text:

Probatur sic, quia Deus posset capere poenam tertii ${ }^{1}$ diei et eam addere poenae primi $^{2}$ diei, et poenam quarti ${ }^{3}$ diei et eam addere poenae secundi $^{4}$ diei, et sic deinceps, sic anticipando poenas quod in tertio die puniretur poena quinti ${ }^{5}$ diei, et quarto die puniretur poena sexti ${ }^{6}$ diei, et sic deinceps. Et consimiliter argueretur de poena secundi ${ }^{7}$ diei et quarti $^{8}$ diei datura ad primam sine nova generatione alicuius poenae prioris vel intensione poenae prioris nisi per solam additionem poenae posteriorum ad poenam priorem.

Tunc quodlibet punctum ${ }^{9}$ in A manet post rarefactionem, quia-ut alias probavi legendo Physicam — non esset rationabile vel possibile in motu rarefactionis corrumpere unum punctum et generare alium eiusdem speciei in eodem situ.

3. The form 'Parisius', which occurs in the manuscripts, is replaced with the corrected 'Parisiis', and the variant itself is excluded from the apparatus criticus.

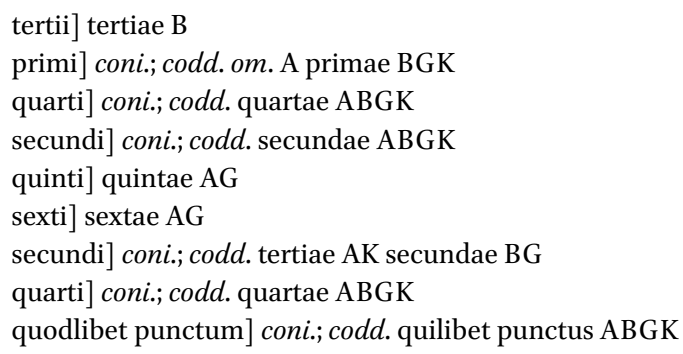


Ad tertium principale, quod quaerit utrum aliquod corpus possit simul et semel esse in diversis locis, haec conclusio conceditur a Monacho Nigro, qui probat quod idem homo numero possit esse Romae et Parisiis simul et semel. Et hoc probavit sic: posito quod Socrates generabit Platonem Parisiis pro A mensura, sic quod in instanti terminante A sit Plato generatus, et sit B terminans A, tunc arguitur sic: quidquid Socrates potest generare pro A mensura Parisiis Deus potest creare ante A per unam diem Romae et ipsum conservare continue per annum Romae sine omni transmutatione vel corruptione posita in aliquo alio, quia aliter Deus indigeret transmutatione et corruptione alicuius extrinseci ex hoc quod crearet.

The division of the text, therein the division into questions, arguments, and responses to arguments, mirrors the original division of the manuscripts. The titles of the articles and dubia are Kilvington's, whereas the subtitles, such as Argumenta pro/contra, Responsiones, and Opinio auctoris, are my own.

The edition observes modern conventions of punctuation. Nonetheless, given the specificity of the text, some explanations are in order. Since the text of Questions on the Sentences abounds in technical terminology and the arrangement of both arguments ('pro' and 'contra') and the responses to the arguments is complex and sometimes difficult to follow, various punctuation marks are employed to facilitate comprehension. Additional numbering of multiple levels of argument (I, 1, A, etc.) is also provided, leaving solely the arguments from authority unnumbered. If a sentence is relatively easy to follow, commas are generally used to separate arguments from conclusions. In longer or more complex sentences, semicolons and em dashes are applied to make the structure of the text more intelligible. For example, Kilvington often strings a long sequence of premises and arguments together, sometimes inserting interpolations, before reaching the conclusion.

Et si arguas contra: videns Socratem Romae et habens visum bene dispositum videret Socratem esse album, et visus non errat circa proprium obiectum, ut patet II De anima commento 63, igitur Socrates foret albus, eadem ratione, videns Socratem Parisiis videret ipsum esse nigrum; et per consequens simul foret albus et niger-hic dico quod licet visus non erret circa proprium obiectum de facto et in maiori parte, tamen in casu illo qui ponitur quod est contra cursum communem, visus bene deciperetur, et hoc contingit aliunde de facto, ut de collo columbae.

Another signature feature of Kilvington's style is observable in quotations. They are composed of multiple excerpts from the quoted works, with 'finis' in 
between them indicating where one portion of the quote finishes and another passage from the source begins. At such points, em dashes are used to separate parts of the quotation. For example:

Septimo: si sic, tunc nullus sacrorum meruit de condigno suam gloriam, et sic non sufficeret ad alios adiuvandos per eorum suffragia. Consequens contra Augustinum XXI De civitate Dei capitulo ultimo: "illi qui recipiuntur" etc.,-_finis—“vita sufficere."

Ad sextum negatur consequentia: 'voluntas bona minus meretur de praemio de condigno vel omnino nihil meretur, igitur est minus bona vel libera etc., quia econtra de facto plus praemiatur voluntas bona quam puniatur mala, quia super illa littera Ad Corinthios 5: non sicut delictum $\langle$ Adae $\rangle$ non est efficax peccatum etc.,-—finis—quam delictum mortificare.

Also, single inverted commas are used to mark a hypothesis, a premise, a consequence, etc., provided that it is preceded by terms such as 'propositio', 'consequentia', etc., but not if it is only introduced by 'it follows'. In the latter case, a colon is employed ('it follows: ...'), or no punctuation is used at all ('it follows that'). For example:

Minor probatur, quia aliter foret haec propositio vera: 'in conceptu amor Dei est Deus vel velle Deum est Deus, quia in conceptu praedicatur idem de se, cum eadem sit intellectio subiecti et praedicati'.

Ad vicesimum sextum dicitur negando consequentiam: 'anima ex naturalibus suis est capax beatitudinis, igitur anima ex puris naturalibus suis potest beatificare se vel esse beata', quia licet ex naturalibus sit passiva et capax beatitudinis passive, non tamen potest se beatificare active nec hoc habet ex naturalibus.

Ibi dico quod non sequitur: tam A quam B infinite ponderant, igitur unum non est gravius alio, quia de infinitis secundum quid unum potest esse maius alio, ut posui in responsione ad 23 argumentum de capacitate animae.

Et sit $\mathrm{C}$ una pedalis quantitas terminata in principio ante rarefactionem ad B situm, et facta rarefactione sequitur quod $C$ numquam erit, quia si $C$ foret infinitae rarefactionis, sequitur quod esset in loco vel situ distante a B situ infinite; sed nullus est nec esse posset talis situs. 
Another feature of Kilvington's style can be observed in phrases addressed to an interlocutor (si velles, si velis, si arguas, sed dices etc.), which is a characteristic feature of the entire commentary, but becomes especially visible in question 3. For example:

Item, glossa ad Philippenses 3: ad ea vero, quae priora sunt, extendens meipsum etc. Sicut enim, si velles implere aliquod subiectum et nosti quod magnum est quod dabitur, extendis subiectum vel sacci vel utris vel alicuius rei, et extendendo facis capaciorem, sic Deus differendo rem extendit desiderium, desiderando extendit animam, et extendendo facit capaciorem. Si igitur Deus extendendo facit animam capaciorem, igitur non fuit infinita.

Et si tu arguas, sicut per partes arguitur in fine huius primi argumenti, quod si aliqua potentia pati posset poenam vel passionem infinitam, igitur, eadem ratione, Deus posset creare aliquam potentiam quae esset simpliciter infinitae potentiae ad agendum, et ita talis potentia esset Deus et creatura aequaretur creatori, quidquid sit de aliis assumptis, potentia talis sic est infinita, igitur est Deus vel aequatur creatori-nego consequentiam.

Et per idem probatur quod talis poena aeterna aequivalet poenis sibi aequalibus, et sic de quacumque volueris, quia subtrahat Deus alternas poenas quas habiturus foret Socrates, et ponat illas per dies continuos in Platone successive, et contiguat poenas alternatas residuas.

Whenever Kilvington provides a series of arguments from authority composed of quotes, they are separated by a semicolon or a comma. For example:

His non obstantibus, dico quod nullus meretur aliquod de condigno de rigore iustitiae, quia Deus non est alicuius debitor nisi forte ex promisso, nos vero ei sumus debitores ex commisso, ut dicit Augustinus De vera innocentia propositione 126: si hoc in nobis est, quod ipsi facimus, inde damnabimur, si autem quod Deus fecit, coronabimur; et III De libero arbitrio 25: nulli Deus debet aliquid, quia omnia gratuite praestat-finisquidquid potest melius esse.

Consequens est falsum et contra Augustinum III De libero arbitrio capitulo 19 et Enchiridion 18: omnis natura, dum corrumpitur, minus bonum est. Aut ei non nocet corruptio et non corrumpitur aut si corrumpitur nocet ei 
corruptio. Et VII Confessionum capitulo 12: Nocet ei corruptio; igitur si in A corrumpitur anima Socratis, tunc nocet ei corruptio.

Confirmatur per Gregorium 19 Moralium capitulo 12: gratia 〈desuper $\rangle$ veniens agit ut digni simus. Et Actuum 5: ibant Apostoli gaudentes quoniam digni etc. Et Sapientia 3: invenit eos dignos se. Et Apocalypsis 3: ambulabunt mecum etc.

Item, Enchiridion 19 et 49: nullus glorietur de voluntatis arbitrio etc. Et Magister II libro distinctione 24 capitulo 14 dicit quod una species superbiae est cum quis credit aliquid sibi esse debitum pro meritis suis. Et Magister II libro distinctione 27 capitulo 5: merita nostra sunt Dei misericordia.

Only direct references and quotations are identified, and only verbatim quotations are placed in double quotation marks. In other instances, the quoted passages are italicized to distinguish them from Kilvington's own text.

Spelling remains a debated issue in critical editions of medieval philosophical and theological texts. In the present edition, orthography is classicized, which means that diphthongs are used wherever classical Latin requires them. In particular, the 'ae' and 'oe' diphthongs replace the 'e' usage of the medieval manuscript practice. Also, 'ci' supplants 'ti', and the Renaissance v/u distinction is implemented throughout. The names of Socrates and Plato appear in their full form, even though the manuscripts give just ' $\mathrm{S}$, 'Sor', and ' $\mathrm{P}$ ' in most cases.

With the exception of 'etc.' no abbreviations are used.

$\begin{array}{ll} & \text { Abbreviations } \\ & \\ ] & \text { scripsit } \\ \langle\ldots\rangle & \text { textus ab editore suppletus } \\ (?) & \text { textus dubius } \\ (!) & \text { sic! } \\ \text { add. } & \text { addidit } \\ \text { al. manu } & \text { alia manu } \\ \text { cap. } & \text { capitulum } \\ \text { codd. } & \text { codices } \\ \text { coni. } & \text { conieci } \\ \text { corr. } & \text { correxit } \\ \text { del. } & \text { delevit } \\ \text { dist. } & \text { distinctio }\end{array}$




$\begin{array}{ll}\text { epist. } & \text { epistula } \\ f . & \text { folium } \\ \text { hom. } & \text { homoeoteleuton } \\ \text { in marg. } & \text { in margine } \\ \text { inv. } & \text { invertit } \\ \text { iter. } & \text { iteravit } \\ \text { lac. } & \text { lacuna } \\ \text { lect. } & \text { lectio } \\ \text { lib. } & \text { liber } \\ n . & \text { numerus } \\ \text { om. } & \text { omisit } \\ \text { p. } & \text { pagina } \\ q . & \text { quaestio } \\ r . & \text { recto } \\ \text { sent. } & \text { sententia } \\ \text { superscr. } & \text { superscripsit } \\ \text { v. } & \text { verso }\end{array}$

\section{Sigla}

A Bologna, Biblioteca Comunale dell'Archiginnasio, A. 985

B Brugge, Stedelijke Openbare Bibliotheek, 188

D Universitäts-und Forschungsbibliothek Erfurt/Gotha-UB Erfurt, Dep. Erf. CA. $2^{\circ}$ 105

F London, British Library, Harley 3243

G Paris, Bibliothèque nationale de France, Lat. 14576

I Praha, Národní Knihovna České Republiky, III B. 10

K Città del Vaticano, Biblioteca Apostolica Vaticana, Vat. lat. 4353

L Wrocław, Biblioteka Uniwersytecka we Wrocławiu, IV F 198

\section{Bibliographical References}

CCSL Corpus Christianorum, series Latina, Turnholti 1953-

CSEL Corpus scriptorum ecclesiasticorum Latinorum, ed. consilio et impensis Academiae Scientiarum Austriacae [olim Academiae Litterarum Caesareae Vindobonensis], Wien 1866-

PL Patrologiae cursus completus, series Latina, accurante J.-P. Migne, Parisiis 1844-1855 et $1862-1865$ 


\section{Alexander Halensis, Summa theologica IIa-IIae}

Alexander Halensis, Summa Fratris Alexandri sive Summa universae theologiaeopus Alexandro moderante conflatum: secunda pars secundi libri (ed. PP. Collegii Sancti Bonaventurae, Quaracchi Firenze: Collegium Sancti Bonaventurae, vol. 3, 1930).

Alexander Monachus, Dicta Anselmi

Alexandri Monachi Cantuariensis Liber ex dictis Beati Anselmi, in Memorials of St. Anselm (ed. R.W. Southern, F.S. Schmitt, Oxford: Oxford University Press, 1969).

Anselmus, Cur Deus homo

Anselmus Cantuariensis, Cur Deus homo, in S. Anselmi Cantuariensis Archiepiscopi Opera Omnia, 6 vols., 1946-1961, vol. 2 (ed. F.S. Schmitt, O.S.B., vol. 1 Seccovii, 1938; vol. 2 Romae, 1940, all reset for Thomas Nelson \& Sons, Edinburgh: Nelson).

Anselmus, De casu diaboli

Anselmus Cantuariensis, De casu diaboli, in S. Anselmi Cantuariensis Archiepiscopi Opera Omnia, 6 vols., 1946-1961, vol. 1 (ed. F.S. Schmitt, O.S.B., vol. 1 Seccovii, 1938; vol. 2 Romae, 1940, all reset for Thomas Nelson \& Sons, Edinburgh: Nelson).

Anselmus, De lib. arb.

Anselmus Cantuariensis, De libertate arbitrii, in S. Anselmi Cantuariensis Archiepiscopi Opera Omnia, 6 vols., 1946-1961, vol. 1 (ed. F.S. Schmitt, O.S.B., vol. 1 Seccovii, 1938; vol. 2 Romae, 1940, all reset for Thomas Nelson \& Sons, Edinburgh: Nelson).

Anselmus, Monologion

Anselmus Cantuariensis, Monologion, in S. Anselmi Cantuariensis Archiepiscopi Opera Omnia, 6 vols., 1946-1961, vol. 1 (ed. F.S. Schmitt, O.S.B., vol. 1 Seccovii, 1938; vol. 2 Romae, 1940, all reset for Thomas Nelson \& Sons, Edinburgh: Nelson).

Anselmus, Proslogion

Anselmus Cantuariensis, Proslogion, in S. Anselmi Cantuariensis Archiepiscopi Opera Omnia, 6 vols., 1946-1961, vol. 1 (ed. F.S. Schmitt, O.S.B., vol. 1 Seccovii, 1938; vol. 2 Romae, 1940, all reset for Thomas Nelson \& Sons, Edinburgh: Nelson).

Aristoteles, De anima

Aristoteles, De anima. Translatio 'noua', Guillelmus de Morbeka, in Sancti Thomae de Aquino Opera omnia, vol. XLV, 1: Sentencia libri de anima (Roma: Commissio Leonina, 1984).

Aristoteles, Met.

Aristoteles, Metaphysica Lib. I-XIV. 4 (ed. G. Vuillemin-Diem, AL XXV. 3, pars secunda, Leiden-New York-Köln: Brill, 1995).

Aristoteles, Phys.

Aristoteles, Physica. Translatio vetus (ed. F. Bossier, J. Brams, AL VII.1.2, Leiden-New York: Brill, 199o).

Augustinus, Conf.

Augustinus Hipponensis, Confessionum libri tredecim (ed. L. Verheijen, CCSL 27, 1981). 
Augustinus, Contra Iulianum

Augustinus Hipponensis, Contra Iulianum (PL 44).

Augustinus, De civ. Dei

Augustinus Hipponensis, De civitate Dei (ed. B. Dombart, A. Kalb, CCSL 47-48, 1955).

Augustinus, De correp. et gratia

Augustinus Hipponensis, De correptione et gratia (PL 44).

Augustinus, De divers. quaest. 83

Augustinus Hipponensis, De diversis quaestionibus LXXXIII (ed. A. Mutzenbecher, CCSL 44A, 1975).

Augustinus, De Genesi ad litt.

Augustinus Hipponensis, De Genesi ad litteram libri duodecim (ed. J. Zycha, CSEL 28/1, 1894).

Augustinus, De lib. arb.

Augustinus Hipponensis, De libero arbitrio (ed. W.M. Green, CCSL 29, 1970).

Augustinus, De musica

Augustinus Hipponensis, De musica (ed. M. Jacobsson, CSEL 102, 2017).

Augustinus, De nat. boni

Augustinus Hipponensis, De natura boni (ed. J. Zycha, CSEL 25, 1891).

Augustinus, De sermone Domini in monte

Augustinus Hipponensis, De sermone Domini in monte (ed. A. Mutzenbecher, CCSL $35,1967)$.

Augustinus, De Trin.

Augustinus Hipponensis, De Trinitate libri XV (ed. W.J. Mountain, CCSL 50-50A, 1968).

Augustinus, De vera religione

Augustinus, De vera religione (ed. K.D. Daur, CCSL 32, 1962).

Augustinus, Ench.

Augustinus Hipponensis, Enchiridion de fide, spe et caritate (ed. E. Evans, CCSL 46, 1969).

Augustinus, Epistulae

Augustinus Hipponensis, Epistulae (ed. A. Goldbacher, CSEL 34/1; 34/2; 44; 57; 58, 1895-1898).

Augustinus, In Iohannis evangelium tractatus

Augustinus Hipponensis, In Iohannis evangelium tractatus (ed. R. Willems, CCSL 36 , 1954).

Augustinus, Retr.

Augustinus Hipponensis, Retractationum libri II (ed. P. Knöll, CSEL 36, 1902).

Ps. Augustinus, De salutaribus documentis ad quemdam comitem

Ps. Augustinus, De salutaribus documentis ad quemdam comitem (PL 40). 
Ps. Augustinus, De spiritu et anima

Alcherus Claraevallensis (dubium) (= Augustinus [pseudo]), De spiritu et anima (PL 40).

Ps. Augustinus, De vera et falsa poenitentia

Ps. Augustinus, De vera et falsa poenitentia (PL 40).

Averroes, De substantia orbis

Averroes, De substantia orbis (transl. Michael Scotus. Revised digital copy of Alvaro de Toledo, Commentario al "De substantia orbis" De Averroes (Aristotelismo y Averroismo), ed. Manuel Alonso, Madrid: Bolaños y Aguilar, 1941. Cologne: Digital Averroes Research Environment (DARE), 2012. URI: www.dare.uni-koeln.de/app/sourcevie wer?type=text \&textid=25 accessed 24.04.2020).

Averroes, In De anima

Averroes, Commentum libri de anima (transl. Michael Scotus. Digital copy of Averrois Cordubensis Commentarium magnum in Aristotelis De anima libros, Corpus Commentariorum Averrois in Aristotelem. Versio latina 6, 1, ed. F. Stuart Crawford, Cambridge, MA: Mediaeval Academy of America, 1953. Cologne: Digital Averroes Research Environment (DARE), 2011. URI: www.dare.uni-koeln.de/app/sourcevie wer?type $=$ text $\&$ textid $=33$ accessed 24.04.2020).

Averroes, In De coelo et mundo

Averroes, Commentum super libro de coelo et mundo (transl. Michael Scotus. Digital copy of Averrois Cordubensis commentum magnum super libro De celo et mundo Aristotelis, ed. F. Carmody, R. Arnzen, Recherches de Théologie et Philosophie médiévales. Bibliotheca 4, Leuven: Peeters, 2003. Cologne: Digital Averroes Research Environment (DARE), 2012. URI: www.dare.uni-koeln.de/app/sourceviewer?type=text \&textid $=23$ accessed 24.04.2020).

Averroes, In Met.

Aristotelis Metaphysicorum libri XIV cum Averrois Cordubensis in eodem commentariis, in Aristotelis Opera cum Averrois Commentariis, vol. VIII (Venetiis apud Junctas 1562$)$.

Beda, Expositio actuum apostolorum

Beda, Expositio actuum apostolorum (ed. M.L.W. Laistner, CCSL 121, 1983).

Bernardus Claraevallensis. Liber de praecepto et dispensatione

Bernardus Claraevallensis, Liber de praecepto et dispensation, in Bernardi opera, 1957-1977, vol. 3 (ed. J. Leclercq, H.M. Rochais, Rome: Editiones Cistercienses, 1963, pp. 253-294).

Biblia Sacra vulgatae editionis (Sixti V Pont. Max. iussu recognita et Clementis VIII auctoritate ed., cura et studio Monachorum Abbatiae Pontificiae Sancti Hieronymi in Urbe Ordinis Sancti Benedicti).

Actus Apostolorum (Act)

Apocalypsis (Apc) 
Deuteronomium $(D t)$

Ecclesiastici $(E c l)$

Iacobi (Iac)

Ioannes (Io)

Iob (Iob)

Lucas $($ Lc)

Mattheus (Mt)

Marcus (Mc)

Philippenses (Phil)

Proverbia (Prv)

Sapientia (Sap)

Boethius, De Trinitate

Boethius, Quomodo trinitas unus Deus ac non tres dii (ed. H.F. Stewart, E.K. Rand, S.J. Tester, The Theological Tracts; The Consolation of Philosophy, and ed. Cambridge, MA-London, 1973).

Clemens V, Constitutiones

Clementis Papae V Constitutiones (ed. Ae.L. Richter, Ae. Friedberg, in Corpus Iuris Canonici, ed. Ae.L. Richter, Ae. Friedberg, 2 vols., Lipsiae 1879/1881, photomech. Nachdr. Graz, 1959).

Decretum magistri Gratiani

Decretum magistri Gratiani (ed. Ae.L. Richter, Ae. Friedberg, in Corpus Iuris Canonici, ed. Ae.L. Richter, Ae. Friedberg, 2 vols., Lipsiae 1879/1881, photomech. Nachdr. Graz, 1959).

Duns Scotus, Ordinatio

Ioannes Duns Scotus, Ordinatio, in Ioannis Duns Scoti Opera omnia (ed. Vaticana, Città del Vaticano, 1950 sqq., vol. VI, 1956; vol. VII, 1973; vol. XIV, 2013).

Gregorius IX, Decretales

Gregorius Papa IX, Decretales (ed. Ae.L. Richter, Ae. Friedberg, in Corpus Iuris Canonici, ed. Ae.L. Richter, Ae. Friedberg, 2 vols., Lipsiae 1879/1881, photomech. Nachdr. Graz, 1959).

Gregorius Magnus, Moralia in Iob

Gregorius Magnus, Moralia in Iob (ed. M. Adriaen, CCSL 143-143A, 1979).

Henricus de Gandavo, Quodl. IV

Henricus de Gandavo, Quodlibet IV, q. 9: Utrum aliquis intellectus creatus ex puris naturalibus possit videre seu intelligere nude divinam essentiam (ed. G.A. Wilson, G.J. Etzkorn, Ancient and Medieval Philosophy. Series 2, Henrici de Gandavo Opera Omnia 8, Leuven: University Press, 2011).

Henricus de Gandavo, Quodl. V

Henricus de Gandavo, Quodlibet V, Paris, Bibliothèque nationale de France, Lat. 15848: 
q. 3: Utrum in Deo sit ponere aliquam infinitatem idearum vel cognitorum, $\mathrm{ff}_{1} 12 \mathrm{O}^{\mathrm{vb}}$ $122^{\mathrm{vb}}$;

q. 20: Utrum adultus sine gratia, quae est quasi habitus generalis ad omnes infusos, possit cavere peccatum mortale, ff. $154^{\mathrm{rb}}-156^{\mathrm{vb}}$.

Henricus de Gandavo, Quodl. V

Henricus de Gandavo, Quodlibet V, q. 35: Utrum filius possit a Deo puniri pro peccato patris, Henricus Gandavensis, and Jodocus Badius. Quodlibeta Magistri He[n]rici Goethals a Gandauo Doctoris Sole[n]nis: Socii Sorbonici \& Archidiaconi Tornacen [sis]. Cum Duplici Tabella. [Parisiis]: Vænundantur ab Iodoco Badio Ascensio, 1518, ff. 211 ${ }^{\mathrm{r}}-$ $212^{\mathrm{r}}$.

Hieronymus, Contra Vigilantium

Hieronymus, Contra Vigilantium (PL 23).

Hugo de Sancto Victore, De sacramentis christianae fidei

Hugo de Sancto Victore, De sacramentis christianae fidei (PL 176).

Isidorus, Sent.

Isidorus Hispalensis, Sententiae (ed. P. Cazier, CCSL 111, 1998).

Liber de Sancti Anselmi Similitudinibus

Liber de Sancti Anselmi Similitudinibus (PL 159).

Monachus Niger, Quaestiones seu Determinationes

Monachus Niger, Quaestiones seu Determinationes, Brugge, Stedelijke Openbare Bibliotheek, 503:

q. 1: Utrum omnis credens non, sicut ecclesia credit, erret in credendo necessario ad salutem, ff. $106^{\mathrm{ra}}-110^{\mathrm{rb}}$;

q. 2: Utrum circumstantia temporis gratiae ex se causet aliquam bonitatem in opere virtuoso, $\mathrm{ff} .11 \mathrm{O}^{\mathrm{rb}}-115^{\mathrm{vb}}$.

Petrus Lombardus, Collectanea in omnes Pauli apostoli Epistulas (PL 191-192).

Petrus Lombardus, In epist. ad Coloss.

Petrus Lombardus, In epist. ad Philipp.

Petrus Lombardus, In epist. ad Rom.

Petrus Lombardus, In epist. II ad Timotheum

Petrus Lombardus, In Psalmos

Petrus Lombardus, Commentarium in Psalmos (PL 191).

Petrus Lombardus, Sent.

Petrus Lombardus, Sententiae in IV libris distinctae (ed. Collegii S. Bonaventurae, 2 vols., Grottaferrata [Romae], 1971/1981).

Prosper Aquitanus, Liber sententiarum

Prosper Aquitanus, Liber sententiarum (ed. M. Gastaldo, CCSL 68A, 1972).

Ricardus Kilvington, Quaestiones super Physicam

Ricardus Kilvington, Quaestiones super Physicam, q. 7: Utrum corpus simplex movebitur aeque velociter in vacuo et in pleno, Venice, Biblioteca Nazionale Marciana, Lat. VI 72 (2810), ff. $101^{\mathrm{ra}}-107^{\mathrm{vb}}$. 
Richardus de Sancto Victore, De Trinitate

Richardus de Sancto Victore, De Trinitate (ed. J. Ribaillier, Textes philosophiques du Moyen Âge VI, Paris 1958).

Robertus Grosseteste, In Phys.

Roberti Grosseteste, Commentarius in VIII libros Physicorum Aristotelis (ed. R.C. Dales, Boulder, CO: University of Colorado Press, 1963).

Robertus Grosseteste, In Post.

Robertus Grosseteste, Commentarius In Posteriorum Analyticorum Libros (ed. P. Rossi, Firenze: L. Olschki, 1981).

Thomas de Aquino, Catena Aurea in Lucam

Thomas de Aquino, Catena Aurea in Lucam, in Catena Aurea in Ouatuor Evangelia, (ed. P. Angelici Guarienti O.P., Turin: Marietti, 1953).

Thomas de Aquino, In Phys.

Thomas de Aquino, In Aristotelis libros Physicorum (textus Leoninus, II, 1884, ex ed. Marietti, 1954). 